\title{
Modelling and validation of spectral reflectance for the colon
}

\author{
Džena Hidović-Rowe and Ela Claridge \\ School of Computer Science, The University of Birmingham, Birmingham B15 2TT, UK \\ E-mail: D.Hidovic@cs.bham.ac.uk and E.Claridge@cs.bham.ac.uk
}

Received 5 June 2004, in final form 4 January 2005

Published 23 February 2005

Online at stacks.iop.org/PMB/50/1071

\begin{abstract}
The spectral reflectance of the colon is known to be affected by malignant and pre-malignant changes in the tissue. As part of long-term research on the derivation of diagnostically important parameters characterizing colon histology, we have investigated the effects of the normal histological variability on the remitted spectra. This paper presents a detailed optical model of the normal colon comprising mucosa, submucosa and the smooth muscle layer. Each layer is characterized by five variable histological parameters: the volume fraction of blood, the haemoglobin saturation, the size of the scattering particles, including collagen, the volume fraction of the scattering particles and the layer thickness, and three optical parameters: the anisotropy factor, the refractive index of the medium and the refractive index of the scattering particles. The paper specifies the parameter ranges corresponding to normal colon tissue, including some previously unpublished ones. Diffuse reflectance spectra were modelled using the Monte Carlo method. Validation of the model-generated spectra against measured spectra demonstrated that good correspondence was achieved between the two. The analysis of the effect of the individual histological parameters on the behaviour of the spectra has shown that the spectral variability originates mainly from changes in the mucosa. However, the submucosa and the muscle layer must be included in the model as they have a significant constant effect on the spectral reflectance above $600 \mathrm{~nm}$. The nature of variations in the spectra also suggests that it may be possible to carry out model inversion and to recover parameters characterizing the colon from multi-spectral images. A preliminary study, in which the mucosal blood and collagen parameters were modified to reflect histopathological changes associated with colon cancer, has shown that the spectra predicted by our model resemble measured spectral reflectance of adenocarcinomas. This suggests that an extended model, which incorporates parameters corresponding to an abnormal colon, may be effective for differentiation between normal and cancerous tissues.
\end{abstract}




\section{Introduction}

The most common examination method in the diagnosis of gastrointestinal disorders is white light endoscopy. Although it can easily identify advanced cancers, early flat and displastic lesions may be easily missed due to their inconspicuous appearance (Dacosta et al 2002). During the endoscopic examination, biopsy samples are collected from suspicious regions for subsequent histological analysis. This is an invasive procedure and there is considerable risk that a diseased region will be missed. To improve the detection rate, and to provide a non-invasive alternative to biopsy, a range of optical methods have been investigated, including diffuse reflectance spectroscopy, fluorescence spectroscopy, Raman spectroscopy, optical coherence tomography and elastic light scattering spectroscopy (see a recent review by Dacosta et al 2002). Common to all these methods is the need to understand the relationship between tissue histology and measured optical quantities, so that parameters characterizing the tissue can be derived from optical measurements.

To be of diagnostic use, any measurements must be capable of differentiating between normal and diseased tissue. Two key features associated with malignant and pre-malignant changes in colon are increased blood volume, most likely due to neovascularisation, and increased thickness and disorganization of the collagen fibre network and extracellular matrix. These changes are known to affect the diffuse spectral reflectance of the tissue (see section 7.1) and the challenge is to develop suitable interpretation methods relating spectroscopic measurements to the state of colon tissue.

Researchers studying diffuse reflectance spectroscopy have successfully used computational modelling of spectral reflectance as a tool to aid the interpretation of measured spectral data. Spectral reflectance models have been developed for a number of human tissues, for example the skin (Cotton and Claridge 1996, Meglinski and Matcher 2002) and the eye (Delori and Pflibsen 1989, Hammer and Schweitzer 2002, Preece and Claridge 2002). However, little work has been reported on colon modelling.

The key contributions in this area come from a group including Backman, Feld, Perelman, Zonios and others, who modelled the spectral reflectance of the colon by means of theoretical models of light propagation in tissue e.g. (Perelman et al 1998, Zonios et al 1999, Badizadegan et al 2004). In their work, light transport was modelled using the diffusion approximation (Farrell et al 1992), and the colon tissue was assumed to be a 'homogeneous semi-infinite turbid medium' (Zonios et al 1999 p 6629). The advantage of their approach was that the solution to the light transport equation, i.e. the derivation of tissue parameters from measured spectra, could be found analytically. This was, however, achieved at the cost of simplifying both the light interaction model and the model of the colon tissue. Georgakoudi et al (2003) suggested that two aspects of that work might benefit from improvement. First, the small distance between the light delivery and collection fibres in the spectral probe used in the data acquisition, made the use of the diffusion approximation inappropriate (Mourant and Bigio 2003) and, ideally, a more accurate light transport model should be used. Secondly, the simplification of the colon architecture, so that it comprises only a single layer, leads to a model which is unable to untangle the effects of different tissue layers on the remitted spectrum. The application of such a model to the interpretation of the measured spectra may result in impoverished conclusions and imprecise measurements.

In this work we have developed a detailed optical model of the normal colon comprising three layers, each with different histology. We have further demonstrated that light incident on the colon surface interacts with all three layers, and that no additional layers need to be included because any light which passed through the first three layers has only negligible effect on the remitted spectrum. Diffuse spectral reflectance was modelled using the Monte 


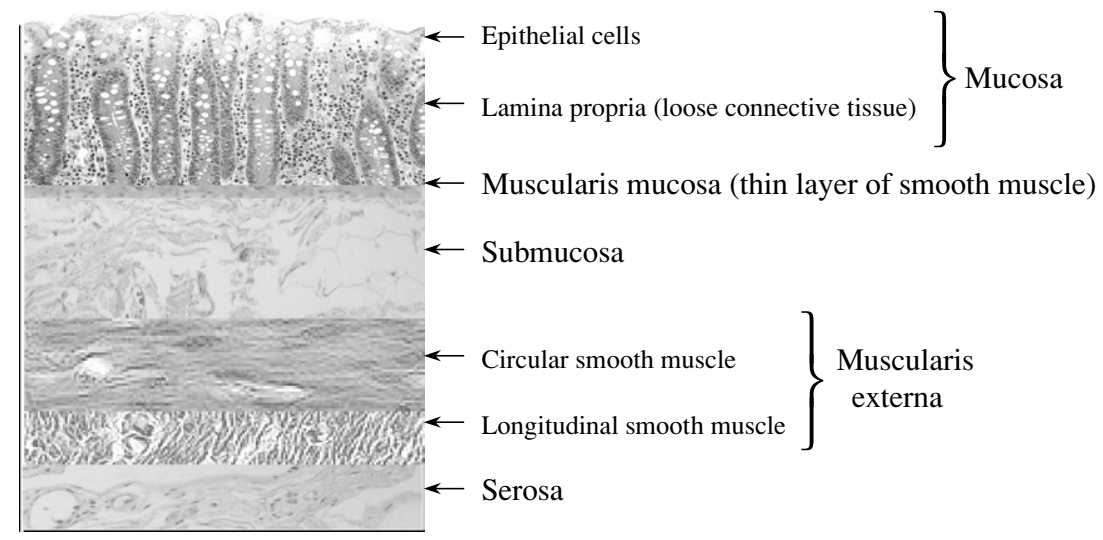

Figure 1. Schematic representation of colon architecture.

Carlo method (Wang et al 1995), which should model light transport in tissue more accurately than the diffusion approximation (Jacques et al 2003). The computed spectral reflectance curves were validated against measured data and their relationship with colon histology was analysed to identify the histological components which most affect the endoscopic appearance of the colon. This work constitutes the first stage of longer-term research whose objective is to derive semi-quantitative information characterizing diagnostically important histological components from multi-spectral endoscopic images.

In this paper, after a brief review of the colon tissue structure in section 2, a detailed explanation of the model of colon tissue colouration, its parameters and their values, is given in section 3. The effects of individual parameters on the total remitted spectra is analysed in section 4 and a simplified version of the model presented. Validation of the model, carried out by comparing its output with the spectra measured from the real tissue, is described in section 5. Good agreement is obtained for the spectra, and, importantly, the parameters used to simulate them are histologically plausible, as presented in section 6 . A discussion on extending the model to include cancerous tissue and of a possible application of the model in a novel method of image interpretation concludes the paper.

\section{Background-light interaction with the colon}

A reflectance spectrum of the colon depends on the tissue structure, its optical properties and the quantities of pigments present in it. Therefore, modelling of the image formation process in the colon requires that this structure is well understood.

Colon tissue is composed of four layers (figure 1) characterized by different optical properties. Starting from the innermost layer, sequentially they are: mucosa, submucosa, muscularis externa propria and serosa. White light incident on the surface of the colon tissue first interacts with the epithelium layer, composed of a one-cell layer of the epithelial cells. The nuclei of the epithelial cells have higher refractive index than the surrounding cytoplasm, and hence act as light scatterers. The size of the nuclei in the normal epithelium is 4-7 $\mu \mathrm{m}$ (Perelman et al 1998), which means that the light is strongly forward scattered. In particular, using the Mie theory (Prahl 2000) we have estimated that the anisotropy factor for a diameter of $5 \mu \mathrm{m}$ varies from 0.982 to 0.99 in the range $400-700 \mathrm{~nm}$. Together with the fact that the epithelium is a relatively thin layer (about $25 \mu \mathrm{m}$ in thickness), this implies that the great 
majority of the light incident on the surface of colon gets transmitted through the epithelium into deeper layers. This is in agreement with what was suggested in previously published reports, e.g. Zangaro et al (1996) report that only a small fraction of the light (2-5\%) reflected back at tissue surface is due to the photons scattered at the cell nuclei of the epithelium layer lining the tissue. The inclusion of the epithelium layer in the colon model would therefore not significantly change the reflectance spectra of the colon tissue.

White light penetrating into the mucosal layer is scattered by a loose network of fine collagen fibres and subcellular organelles, such as mitochondria. The small size of these structures (whose diameter is a fraction of a micron) causes the light from the mucosa to be scattered at small angles, i.e. to be highly back scattered. Within the mucosa, there exists a network of small capillaries which are organized in honeycomb-like networks around the mucosal glands throughout all the length of the colon (Skinner and O'Brien 1996). Capillaries provide red blood cells containing both oxy- and deoxy-haemoglobin which act as a strong light absorber in the visible range of light. Collagen fibrils and subcellular organelles are assumed to be the most important scatterers, while haemoglobin derivatives represent the most important absorbers of the visible light in this layer.

After traversing the mucosa, a small fraction of light is reflected at the boundary of the submucosa due to a change in the refractive index, whilst most penetrates into the submucosa. The submucosa is composed almost entirely of a dense network of larger collagen fibres (Thomson et al 1986). The diameter of these collagen fibres is typically a few microns, which makes this layer a strongly forward scattering medium. Large blood vessels (arteries and veins) supply the submucosa with both oxy- and deoxy-haemoglobin, which causes further absorption of light in this layer. The total amount of blood in the submucosa is greater than that in the mucosa, resulting in an increase in light absorption.

The fraction of the incident light that is not absorbed or back scattered in the first two layers of the colon tissue enters the muscle layer where it gets further strongly absorbed by blood and scattered. The anisotropy factor of this layer is 0.96 (Marchesini et al 1994), meaning that the scattering, once again, is strongly forward directed. Most of the light that penetrates into this layer does not return to the tissue surface. However, a fraction of photons in the red region, which penetrate deeper into the tissue, do re-emerge at the surface, altering the red end of the spectrum.

Finally, the light transmitted through the muscle layer into the deeper layers of colon tissue forms just a very small fraction of the incident light and is mainly confined to the red region of spectrum. It does not significantly affect the light remitted from the colon. This suggests that the spectral reflectance, and thus the colour of colon tissue, depends only on the light interaction with the first three layers. Therefore, our model of colon tissue comprises mucosa, submucosa and muscle layer.

\section{Modelling the spectral reflectance with Monte Carlo method}

In this section, a detailed description of the model of colon reflectance will be given. All the parameters included in the model will be explained, and the origins of their values will be discussed.

\subsection{Modelling scattering and absorption coefficients}

To model the interaction of light with colon tissue we have used the Monte Carlo method (Wang et al 1995), which is considered to be an accurate solution to the radiative transfer equation describing light propagation in the tissue. It requires information about the absorption 
and scattering coefficients of each of the layers composing the model, their respective thicknesses, refractive indexes and anisotropy factors.

Previous literature reports very little on the quantitative optical properties of colon tissue, and specifically on its scattering and absorption coefficients. Marchesini et al (1994) and Zonios et al (1996) both report the optical properties of colon tissue measured ex vivo, i.e. on excised tissue samples. As argued by Marchesini et al (1994), when measured ex vivo, scattering and absorption coefficients are not representative of what is happening in tissue in vivo because the excision of a tissue sample results in changes in the amounts of components, such as, for example, water and blood. Other sources, e.g. Beek et al (1997) and Tuchin (2000), report optical properties of colon at single wavelengths only, and in particular at the near infrared wavelengths which are outside the range of our interest. For these reasons it was necessary to model the scattering and absorption coefficients of mucosa and submucosa by means of theoretical models.

The scattering coefficient can be expressed as

$$
\mu_{s}(\lambda)=\sigma_{s} \rho_{s}(\lambda),
$$

where $\lambda$ is the wavelength, $\sigma_{s}$ is the density of the scattering centres and $\rho_{s}$ is the scattering cross-section which depends on the size and shape of scattering particles, refractive index of the particles and the medium and wavelength.

The density of the scattering centres,

$$
\sigma_{s}=V_{s} \frac{1}{\frac{4}{3} r^{3} \pi},
$$

depends on $V_{s}$, the volume fraction of the scattering particles, and $\frac{1}{\frac{4}{3} r^{3} \pi}$, the maximum number of the scattering particles with radius $r$, assuming that they occupy all the medium.

Under the assumption that the scattering particles are homogeneous spheres, the scattering cross-section can be calculated using the Mie theory (Bohren and Huffman 1983). This assumption is not strictly satisfied in colon as some of its scattering particles have highly non-spherical shape. However, recent studies by Chen et al (2004) suggest that modelling scattering cross-section of non-spherical particles by homogeneous spheres is a plausible approximation (see 7.1).

It should be noted that the modelling of the scattering coefficients has been carried out using a commonly used simplification under which the sizes, the shapes and the densities of the scattering particles are assumed to be the same within a given layer. This is obviously not the case in the real tissue, and particularly in the mucosa, where scattering is affected by collagen fibrils as well as by other organelles. The current model uses mucosal parameters derived from the properties of the collagen fibrils. In normal mucosa the fibril sizes are similar to those of the organelles and are of the order of a fraction of a micron. Their relative refractive index ranges are also similar: organelles 1.03-1.06 (Fang et al 2003), and collagen around 1.06. Due to this simplification, any scatterer quantities reported subsequently in this paper must be taken to represent the mean values for all the contributing particles for a given layer.

The major absorbers of visible light in the colon tissue are oxy- and deoxy-haemoglobin. The absorption coefficient was therefore calculated using a known expression for the absorption by blood (Jacques 1998, Prahl 1999, Meglinski and Matcher 2002):

$$
\mu_{a}(\lambda)=V_{\mathrm{Hb}} \ln 10 c_{\mathrm{Hb}}\left(\alpha \varepsilon_{\mathrm{HbO}_{2}}(\lambda)+(1-\alpha) \varepsilon_{\mathrm{Hb}}(\lambda)\right)
$$

where $V_{\mathrm{Hb}}$ is the volume fraction of the blood in tissue, $c_{\mathrm{Hb}}$ is the concentration of haemoglobin per litre of blood, $\alpha$ is the haemoglobin saturation, and $\varepsilon_{\mathrm{HbO}_{2}}$ and $\varepsilon_{\mathrm{Hb}}$ are the molar extinction of oxyhaemoglobin and haemoglobin (Prahl 1999). The concentration of haemoglobin per unit 
volume of blood in the colon is assumed to be $120 \mathrm{~g} \mathrm{~L}^{-1}$, as suggested by Hajivassiliou et al (1998); according to Prahl (1999), normal haemoglobin concentration in tissue is $150 \mathrm{~g} \mathrm{~L}^{-1}$.

\subsection{Model parameters and their origins}

The Monte Carlo modelling method used in this work requires five parameters for each layer, namely absorption and scattering coefficients, refractive index, anisotropy factor and layer thickness. However, as scattering and absorption coefficients were not directly available for mucosa and submucosa, they had to be themselves modelled from simpler data. This has increased the overall number of parameters necessary for modelling the spectral reflectance of the colon, resulting in the following list: volume fraction of blood and haemoglobin saturation, characterizing light absorption; mean size and volume fraction of the scattering particles, characterizing the scattering of light; and layer thickness. These parameters were defined for both the mucosa and submucosa. For each of the parameters a range of plausible values was defined. Additionally, two constant parameters were defined for the first two layers of the model. Those parameters are the refractive index of the medium and of the scattering particles. Anisotropy factors for mucosa and submucosa were estimated from other parameters using the Mie theory. The muscle layer was modelled using single scattering and absorption curves, and fixed thickness, refractive index and anisotropy factor, as described later in this section.

To our knowledge, a comprehensive set of optical parameters characterizing the normal colon tissue has not been presented before and this constitutes one of the contributions of this paper. Some parameters were not previously reported in the literature, and for some the values reported in the literature varied considerably, as discussed below. Where histological parameter values were not available from the published sources, we derived parameter values through modelling and from measurements using stained images of colon.

\subsubsection{Mucosa}

Volume fraction of blood. Skinner et al (1995) and Skinner and O'Brien (1996) measured the sizes and volume fraction of the blood vessels in human colon by means of electron microscopy. They have reported the following volume fractions of blood vessels: proximal colon $13.4 \pm$ $3.0 \%$; midcolon $12.4 \pm 5.1 \%$; distal colon $7.7 \pm 2.2 \%$. However, it was observed that the difference in the volume fractions of blood vessels between proximal and mid colon was not significant, but only numerical. Therefore we treated both fractions as belonging to the range reported for mid colon. Given that the blood vessels in the real tissue are full of blood, we have used a simple method for extracting the volume fraction of blood from these data. In particular, the volume fraction occupied by the vessel walls was subtracted from the total volume fraction of the vessels, resulting in the following ranges of blood volume fractions: proximal and mid colon 5-15.5\%; distal colon 3.57-8.96\%. In significant disagreement with these findings are values published by Zonios et al (1999) who reported the haemoglobin concentration in the colon mucosa to be $13.6 \pm 8.8 \mathrm{mg} \mathrm{dL}^{-1}$. Given that in the expression for calculating the absorption by haemoglobin they do not explicitly distinguish the volume fraction of blood and the haemoglobin concentration per unit volume in tissue (as in expression 3), we understood that their haemoglobin concentration is actually the product of these two quantities. According to Hajivassiliou et al (1998), the concentration of haemoglobin per unit volume of blood in the colon has a value of $120 \mathrm{~g} \mathrm{~L}^{-1}$. This implies that the volume fraction of blood used by Zonios et al (1999) was $0.04 \%-0.18 \%$. Compared to the findings of Skinner and O'Brien (1996), 
these values seem to be 1-2 orders of magnitude smaller. Furthermore, Zonios et al (1999) give no reference which confirms that their values of haemoglobin concentration are histologically plausible. Based on the balance of all the above evidence we have decided to use the values reported by Skinner and O'Brien (1996). However, in their work on skin, Meglinski and Matcher (2002) reported: 'the volume fraction of dermis occupied by blood vessels is usually in the range 1-20\%, and varies in apparent blood content in the range $2-12 \%$ '. This suggests that the volume fraction of blood in the tissue can be significantly smaller than that of the blood vessels. We have therefore set the lower boundary of values for this parameter to be $2 \%$, and the upper one to $10 \%$.

Haemoglobin saturation. Friedland et al (2003) have measured the mucosal capillary haemoglobin oxygen saturation in the colon by reflectance spectrophotometry. They have reported that the haemoglobin saturation in the colon mucosa has the value $72 \pm 3.5 \%$. Zonios et al (1999) reported haemoglobin saturation of normal mucosa to be $59 \pm 0.08 \%$. Yet another estimate of this parameter in normal mucosa comes from Ge et al (1998). Their statistical analysis of the spectral absorbance resulted in the following approximation: $49 \pm 15 \%$. In our model the range $50-75 \%$ was used.

Collagen size. Zonios et al (1999) reported that sizes (diameters) of scattering particles including collagen in the colonic mucosa vary in the range $0.35-0.74 \mu \mathrm{m}$. However, different values have been reported by Furuya and Ogata (1993), who studied the collagen networks of normal mucosa, hyperplastic polyps, tubular adenoma and adenocarcinoma of the human large intestine by means of electron microscopy and report that in the normal mucosa 'the sub-basal laminar collagen sheath was made up of densely arranged collagen fibrils about $100 \mathrm{~nm}$ in diameter'. To account for both sources, the mean size of collagen fibrils was taken to vary in the range $0.1-0.74 \mu \mathrm{m}$.

Volume fraction of collagen. We were unable to find the value of this parameter in the published literature. Our estimate, derived by the image analysis from microscopy images of histological samples of mucosa stained for collagen (at $\times 400$ magnification), was that the volume fraction of the collagen fibrils is in the range 4-20\%. Furthermore, this range of values used in the modelling of spectra has generated spectral reflectance consistent with the measured spectra.

Thickness. The thickness of the mucosal layer of the colon varies in the range 395-603 $\mu \mathrm{m}$. This range was originally reported by Marchesini et al (1994), and it agrees with the recent findings of Huang et al (2004).

Anisotropy factor. The anisotropy factor was calculated by the Mie theory starting from the information on the collagen size and density (Bohren and Huffman 1983). Given the small sizes of the scattering particles in mucosa, this parameter was found to be in the range $0.50-0.95$.

Refractive index of the medium. This parameter was set to the value 1.38 as suggested by Tuchin (2000). The value was assumed constant for all the wavelengths in the visible range.

Refractive index of collagen fibrils. The refractive index of collagen fibrils was assumed to have the value 1.46 as suggested by Jacques (1996).

\subsubsection{Submucosa}

Volume fraction of blood. The literature does not report an exact value of this parameter for submucosa. However, it is known that submucosa has bigger blood vessels than mucosa and a larger total blood content. Therefore, an assumption was made that the volume fraction 
of the blood in this layer is in the range 5-20\%. This rough estimate was deemed sufficient in the light of computational experiments showing that small changes to this value do not significantly affect the remitted spectrum.

Haemoglobin saturation. The range of values was taken to be the same as for haemoglobin saturation in mucosa, i.e. $50-75 \%$. This seems to be a reasonable assumption, given that mucosa and submucosa have the same blood supply.

Collagen size. In contrast to the mucosa, which has very fine collagen fibrils, the submucosa is packed with collagen fibres of a larger diameter. Ge et al (1998) reported that collagen fibres of normal mucosa vary in the range $2-3 \mu \mathrm{m}$. We have extended this range to $1-6 \mu \mathrm{m}$, to account for observations made during the analysis of stained images of colonic submucosa.

Volume fraction of collagen. As in mucosa, the value of this parameter could not be found in the literature. Based on our image analysis of histological samples stained for collagen, the volume fraction of the collagen fibrils was estimated to be in the range 15-60\%. This range yielded plausible modelled spectra.

Thickness. Thickness of the normal submucosa (including the muscularis mucosa) is in the range $415-847 \mu \mathrm{m}$. This was originally reported by Marchesini et al (1994), and it agrees with the recent findings of Huang et al (2004).

Anisotropy factor. The anisotropy factor of the submucosa is calculated by the Mie theory given the size and density of collagen fibres. Consistent with the large sizes of the scattering particles in mucosa, this parameter was in the range $0.95-0.99$, indicating strongly forward oriented scattering of light.

Refractive index of the medium. This parameter is set to the value 1.36, as suggested by Tuchin (2000), and is assumed to be wavelength independent in the visible range.

Refractive index of collagen fibres. Jacques (1996) argued that collagen fibres have smaller refractive index than collagen fibrils because the water content in the former lowers the corresponding refractive index. Given that the submucosa contains mainly large collagen fibres, a value of 1.38 was used as suggested by Jacques (1996).

3.2.3. Muscle Layer. The muscularis externa, unlike the mucosa and submucosa, is modelled with single scattering and absorption curves. This was considered sufficient, as the light which penetrates the first two layers and enters the muscle layer forms just a very small part of the incident light and is strongly forward directed, with a small fraction of photons in the red end of the spectrum returning to the tissue surface.

Scattering coefficient. Given that all the available literature sources report values of scattering coefficients of the muscle at single wavelengths only, we have applied the exponential model (Mourant et al 1997) to estimate the scattering coefficient for the whole visible range by extrapolation. Using this approach, the reduced scattering coefficient can be approximated as

$$
\mu_{s}^{\prime}(\lambda)=\mu_{s}(\lambda)(1-g)=x \lambda^{c},
$$

where $\lambda$ is wavelength in $\mathrm{nm}, g$ is the anisotropy factor, $x$ is a factor of proportionality and $c$ is a constant exponent. In order to find the values of $x$ and $c$ from the above equation, the values of the scattering coefficient of muscle at two wavelengths were used:

$$
\begin{aligned}
& \mu_{s}(515)=530 \pm 44 \mathrm{~cm}^{-1}, \quad g=0.96 \quad \text { (Marchesini et al 1989) } \\
& \mu_{s}(1064)=238 \mathrm{~cm}^{-1}, \quad g=0.93 \quad \text { (Tuchin 2000). }
\end{aligned}
$$


Using these values to solve the equation 4 , the values of 168.52 and -0.332 are obtained for $x$ and $c$ respectively. Scattering coefficients were then calculated using the equation 4.

Absorption Coefficient. Absorption coefficients for the visible range were obtained by extrapolation from the value of the absorption coefficient at $515 \mathrm{~nm}$ reported by Marchesini et al (1989), using expression (3), which can be rewritten as follows:

$$
\mu_{a}(\lambda)=A\left(\alpha \varepsilon_{\mathrm{HbO}_{2}}(\lambda)+(1-\alpha) \varepsilon_{\mathrm{Hb}}(\lambda)\right),
$$

where $A$ is unknown. Given that it is the only unknown, we can use $\mu_{a}(515)=11.2 \mathrm{~cm}^{-1}$ to extract the value of $A$, and then calculate the absorption coefficients for all the wavelengths in the range 400-700 $\mathrm{nm}$.

This result should be treated with caution for the following reason. Given that Marchesini et al (1989) analysed optics of the muscle tissue ex vivo, the absorption coefficient was affected by the fact that the amount of the blood in muscle was less than in real tissue in vivo. A computational experiment was carried out in which the amount of blood in the muscle was varied, and it was observed that it does not cause big changes in the reflectance spectra of the colon. This could be explained by the fact that only photons in the red region are penetrating the muscle layer. However in that region of the spectrum absorption of blood is very small and hence it does not affect the spectra.

Thickness. Huang et al (2004) reported that the thickness of normal colonic muscle is in the range $600-1010 \mu \mathrm{m}$. Throughout the expriments we have used a fixed value of $900 \mu \mathrm{m}$ to represent the thickness of this layer.

Anisotropy factor. Anisotropy factor was assumed to have a constant value throughout the visible range of wavelengths. The value of 0.96 reported by Marchesini et al (1989) was used in our model.

Refractive index of medium. Refractive index of the medium was set to 1.36 as suggested by Tuchin (2000).

\subsection{Reflectance of the colon using Monte Carlo method}

Having established the model parameters and their histologically plausible ranges, the Monte Carlo method was used to calculate the total diffuse reflectance spectra. Given the high computational complexity of Monte Carlo simulations, which is proportional to the number of wavelengths at which the reflected spectrum is calculated, the spectra were simulated at the following set of 25 wavelengths, chosen to give a sufficiently accurate characterization of the shape of the spectra: $\{400,410,420,430,440,450,480,506,514,522,540,548,560,564$, $568,574,580,586,594,610,620,630,640,676,700\}$. Most wavelengths were selected in the blue and green region of the visible light, because these parts of the spectrum are significantly changed through the light absorption by haemoglobin pigments. Each spectrum was obtained using 200000 photons.

\section{Effect of the individual parameters on the colon spectra}

Figure 2 shows the effects of varying the model parameters on the final remitted spectrum. In each graph, only a single parameter is varied through the corresponding histologically plausible range. All other parameters are set to the mid-values of their ranges. As can be seen, the parameters of the mucosal layer have the greatest influence on the reflectance spectra of the colon. In particular, in the blue and green region, the haemoglobin absorption results 

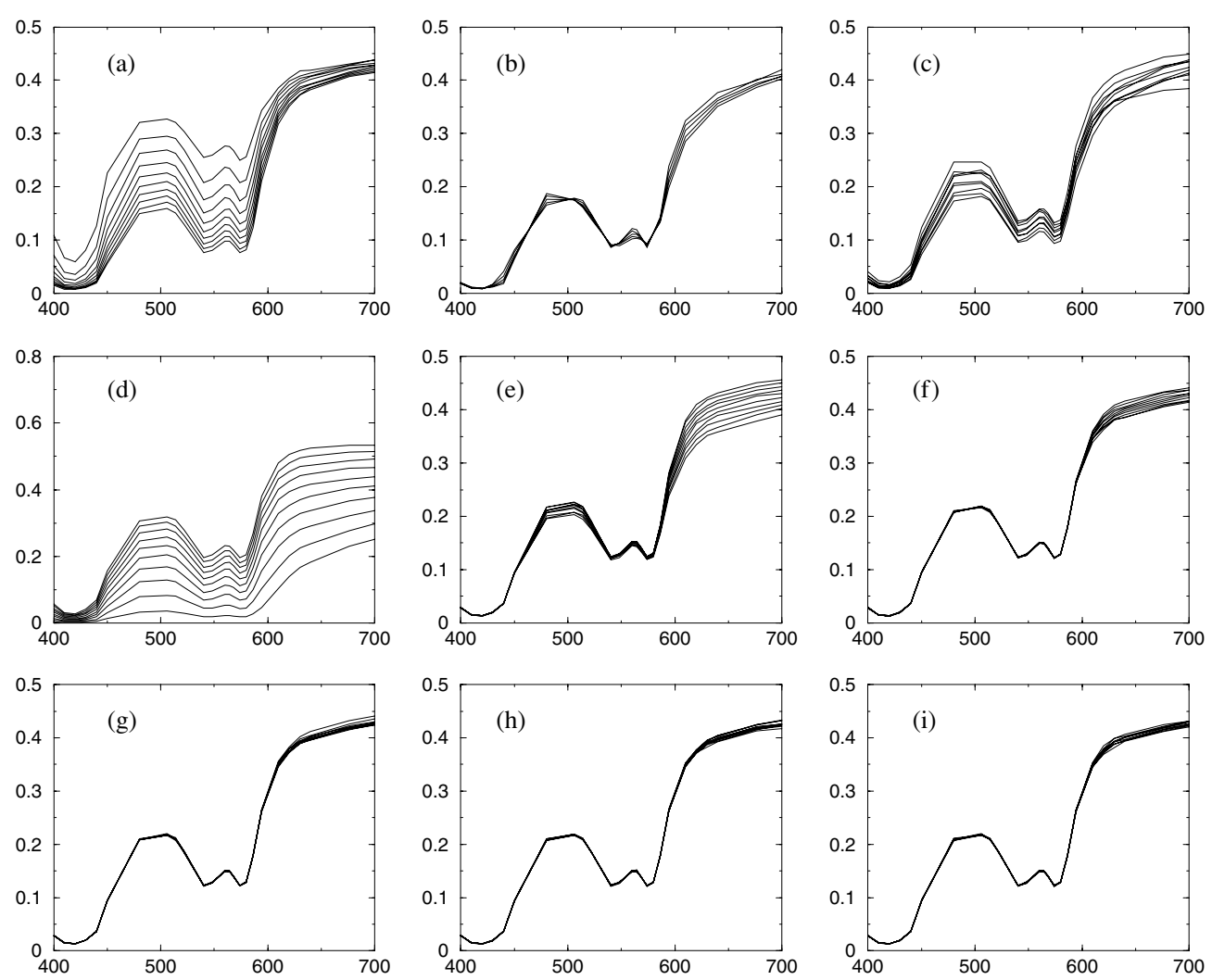

Figure 2. Variations of the colon spectra as a function of varying individual parameters. Parameters were, one at a time, varied in their plausible ranges, while the remaining ones were set to the mean values of the corresponding ranges. Variable parameters were the following: (a) volume fraction of blood in mucosa; (b) haemoglobin saturation in mucosa; (c) size of scattering particles in mucosa; (d) volume fraction of scattering particles in mucosa; (e) thickness of mucosa; (f) volume fraction of blood in submucosa; ( $\mathrm{g}$ ) size of collagen fibres in submucosa; (h) volume fraction of collagen in submucosa; (i) thickness of submucosa (see the text for details).

in the characteristic dips and peaks of the spectrum around $420 \mathrm{~nm}$ and 540-580 nm. They become more prominent with increase in the blood content. Only small changes can be seen in the red part of the spectrum, where the absorption by haemoglobin becomes negligible. A larger blood content also results in smaller remittance signal, as more light gets absorbed on its way inside the tissue. It can be seen from figure 2 that the haemoglobin saturation has a relatively little effect on the spectra in its plausible range.

The size of the scattering particles in mucosa has a very strong effect on the remittance in all parts of the spectrum. The smaller the size, the less forward scattered the light is, and hence the more light is remitted in blue and green, and less in red. Analogously, the converse is true when there is an increase in the diameter of the scattering particles.

The volume fraction of the scattering particles in the mucosal layer causes significant variations in the remitted spectrum through all the wavelength range. The most pronounced changes are in the red region, where absorption by haemoglobins is relatively small, and the remittance is affected mainly by the scattering effect. Changes in the blue and green region are also present as, for a lower volume fraction of the scattering particles, the scattering 

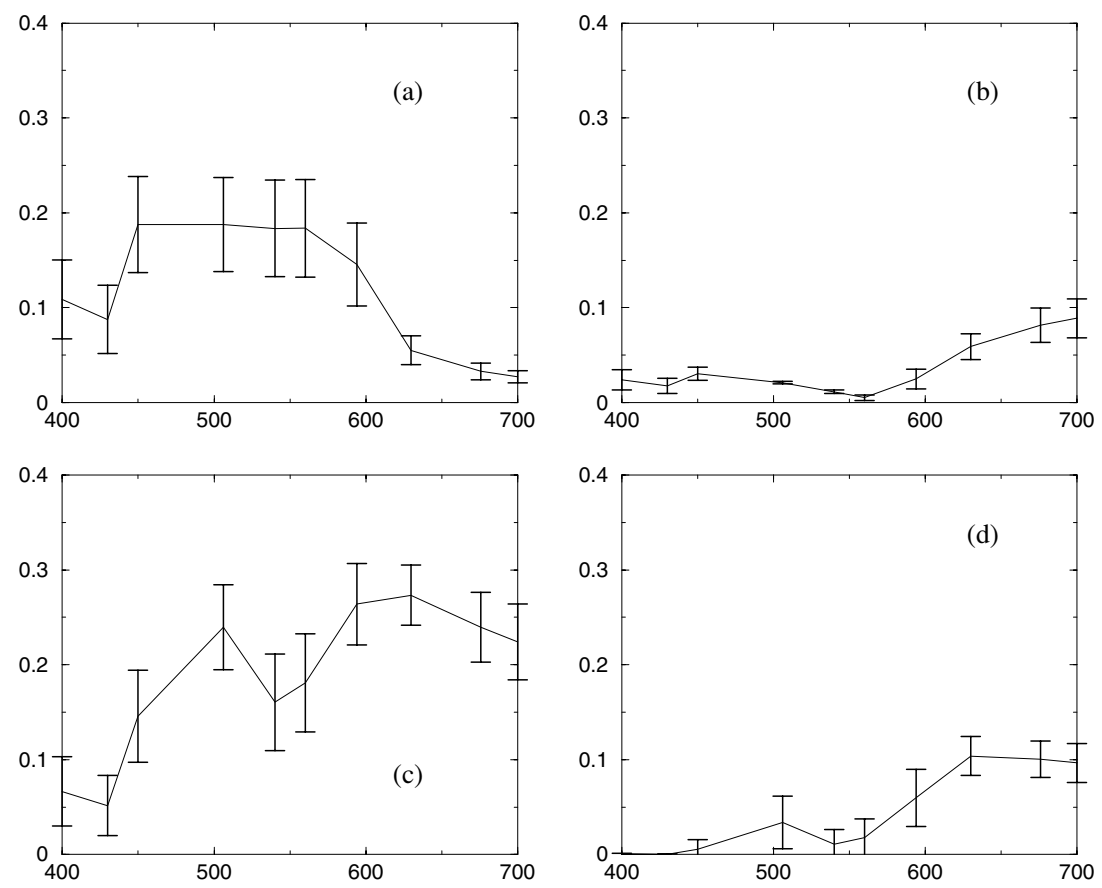

Figure 3. The four graphs show quantitative analysis of the variations of spectra as a function of the variations of the parameter values. The solid line represents the mean value of the spectral variability, with error bars showing the standard deviation for (a) volume fraction of blood; (b) size of scattering particles; (c) density of scattering particles; (d) thickness (see the text for more explanations).

coefficient is smaller and therefore the effect of the haemoglobin absorption on the spectra is greater (Marchesini et al 1994).

In contrast to the mucosa, varying the parameters of the submucosa does not result in big variations of the total reflectance, with changes being limited only to the red region of the spectrum (figure 2). This is as expected, given that photons in the blue and green region are more strongly scattered than those in the red, and hence they probe tissue volume to a lesser depth. While blue and green light travels mostly within the mucosal layer, red light penetrates deeper in the tissue. After being absorbed and scattered, a small part of it then re-emerges at the tissue surface, affecting the changes of the total remitted spectrum.

Given that the mucosal parameters have the most significant effect on the spectra of the colon, we have performed a quantitative analysis of the model's sensitivity to variations in the values of the four most important mucosal parameters, namely blood volume fraction, size of the scattering particles, density of the scattering particles and thickness. To do this, 30 sets of parameter values were randomly chosen from their respective ranges. For each of these sets three parameter values were regarded as fixed, the fourth was replaced by taking its global maximum or minimum value, enabling a pair of spectra to be calculated. This was done for each of the four mucosal parameters. Thus, in all, for each of the 30 sets, four pairs of spectra were computed. Variations of the spectra were then estimated by calculating the difference between the paired spectra at each wavelength, in all 30 cases, and for all four parameters. Mean and standard deviations of the distances were calculated and are presented in figure 3 . Once again, it can be observed that the volume fraction of blood and the scattering particles 

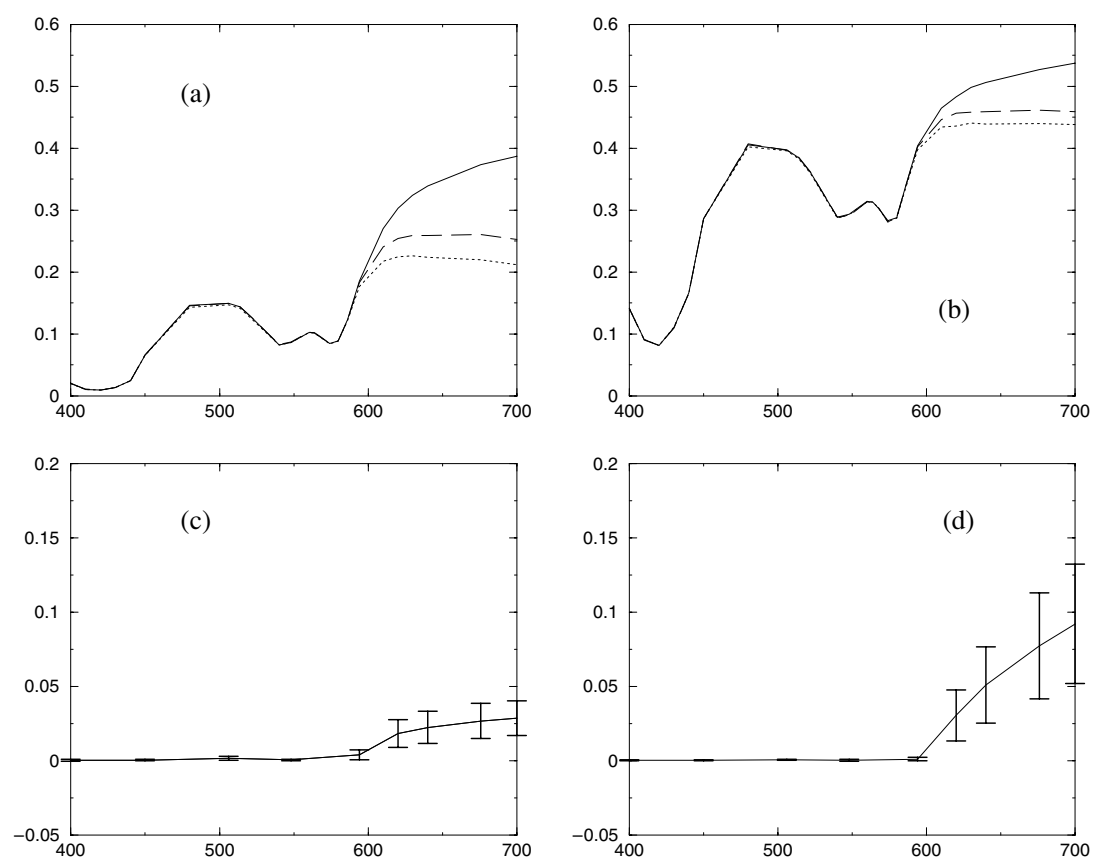

Figure 4. The top two graphs $(a, b)$ show the comparisons of the reflectance spectra of the colon for mucosa only (dotted line); mucosa and submucosa (dashed line); mucosa, submucosa and muscle (solid line); for two randomly chosen combinations of parameter values drawn from their histologically plausible ranges. The bottom two graphs show quantitative analysis of the variations of spectra as a function of the number of layers. The solid line represents the mean value of the spectral variability, with error bars showing the standard deviation for (c) mucosa versus mucosa and submucosa; (d) mucosa and submucosa versus mucosa, submucosa and muscle layer (see the text for more explanations).

in the mucosa have a major impact on the colon spectra. The size of scattering particles and layer thickness have a smaller influence, with the last being confined mainly to the red end of the spectrum.

Although varying the submucosal parameters does not result in significant changes in the final remitted spectrum shapes, this layer, as well as the muscle layer, must be included in the model because their parameters influence the absolute spectral line positions. This observation was confirmed by comparing the spectra reflected from the first layer only with the spectra modelled using two and three layers respectively. In the latter two cases, an increase in the red region of the total remitted spectrum was observed, as shown in figures 4(a) and 4(b) Quantitative analysis of the variations of spectra as a function of the number of layers was carried out by calculating mean and standard deviation of differences between the spectra of only mucosa and spectra of mucosa and submucosa (figure 4(c)); and spectra of mucosa and submucosa versus spectra of first three layers (figure 4(d)) for 20 randomly chosen sets of parameter values. It can be clearly seen that including the second and third layers alters the red end of the spectrum. A further reason for retaining the submucosal and muscle layers is that they may affect the spectrum variability in abnormal colon tissue. This remains a subject of future studies.

Whilst modelling normal tissue, the constant effect of the submucosa on the final spectrum has suggested an obvious simplification of our model. Instead of allowing the parameters of 
Table 1. Values of submucosal parameters used in this paper.

\begin{tabular}{lllll}
\hline $\begin{array}{l}\text { Volume fraction } \\
\text { of blood }\end{array}$ & $\begin{array}{l}\text { Haemoglobin } \\
\text { saturation }\end{array}$ & $\begin{array}{l}\text { Mean collagen } \\
\text { size }\end{array}$ & $\begin{array}{l}\text { Mean volume } \\
\text { fraction of collagen }\end{array}$ & Thickness \\
\hline $20 \%$ & $75 \%$ & $3.5 \mu \mathrm{m}$ & $50 \%$ & $700 \mu \mathrm{m}$ \\
\hline
\end{tabular}

the submucosa to take a range of values, they can be set to a single set of plausible values discussed in the previous section. This makes the model less complex, and hence more easy to analyse and invert. It is clear that this simplification introduces a small error to the spectra generated by our model, but this is acceptable given that a final goal of our work is the estimation of relative and not absolute quantities of the histological parameters. The obvious limitation is that the simplified model permits only extracting the information about specific parameter values of the mucosa. However, this does not necessarily diminish the significance and usefulness of our method, as early changes of the tissue due to development of pathologies and cancer of the colon are confined to the mucosal layer. Hence, being able to characterize only the structure of mucosa would still be of a great value in diagnosis. Therefore, in the remainder of this paper, the submucosal parameters of the model are set to constant values (see table 1), and only those of the mucosa are varied in their appropriate ranges.

\section{Model validation}

The main objective of the validation process was to verify the correctness of the spectral reflectance model of colon. This has been done by comparing spectra computed from the model by Monte Carlo simulations with spectra measured from real tissue. If the model is correct, then it must be able to generate spectra which approximate well the measured spectral reflectances, and the parameters used for modelling must correspond to histologically plausible ranges. However, this is only the first step, as the converse assertion is not necessarily true.

The simulated spectra were compared against two independent sets of the experimental spectra obtained by diffuse reflectance spectroscopy in vivo. The first data set was obtained using a probe composed of 16 fibre optics, each having a diameter of $200 \mu \mathrm{m}$, randomly placed in a circle. Eight fibres were used to deliver the light to the tissue, and another eight to collect the remitted light. The probe was passed through a working channel of an endoscope and placed in contact with the colon wall of the patients during ordinary colonoscopy procedures (Ge et al 1998). Out of 84 spectra of normal colon, kindly provided by Kevin Schomacker, 54 have been used in the validation.

The second set of the data, comprising 50 spectra of normal colon, was collected by Sambongi et al (2002) using a non-contact probe with significantly different geometry than the one described above. The illuminating fibres were placed at the centre of the probe, in a circle of diameter $1 \mathrm{~mm}$. The light receiving fibres were placed around these in a ring, with an outer diameter $2 \mathrm{~mm}$. Probe to tissue distance was approximately $1 \mathrm{~mm}$. The measured spectra were recorded every $2 \mathrm{~nm}$ in the range 300-800 $\mathrm{nm}$ for the first data set, and 400-800 nm for the second data set. No spectra of abnormal colon were used in the validation because the model developed so far simulates the light interaction with normal tissue only.

It is well known that the spectral composition and the intensity of the collected light depend not only on the tissue structure but also on the geometry of the probe used in measurements. Therefore, comparisons between in vivo measured spectra and modelled spectra are meaningful only if the real and the virtual probes collect compatible spectral information. For that reason, 
it was necessary to model the probes used by Ge et al (1998) and Sambongi et al (2002) in their measurements. In particular, the output of the Monte Carlo method which gives the spectral reflectance corresponding to the pencil beam of incident light (Wang et al 1995), was convolved to yield the response to a uniform beam of illumination (Wang et al 1997) generated by the illuminating fibres. This response was then further convolved against the specific distribution of collecting fibres in the probes to yield the total responses of the two probes. In this last step, the numerical aperture of the collecting fibres was taken into account when calculating the amount of remitted light that was actually collected. In addition, for the non-contact probe of Sambongi et al (2002), probe to tissue distance was taken into account.

The validation was implemented as an optimization problem, with the aim of minimizing the distance between the simulated and measured spectra. The distance between the two curves was calculated using the following measure of distance between two sets of points:

$$
d(y, z)=\frac{1}{m} \sum_{i=1}^{m}\left|y_{i}-z_{i}\right|,
$$

where $y_{i}$ and $z_{i}$ are the values of measured and simulated spectral reflectances at the wavelength $w_{i}$, and $m$ is the total number of wavelengths (in our case $m=25$ ).

Simulated spectra were generated using the input parameters described in section 3.2, namely: constants characterizing the refractive index of the medium and scattering particles, and the anisotropy factor for mucosa, submucosa and muscle; fixed parameters characterizing the absorption and scatter of submucosa and muscle; and five variable model parameters describing the mucosa blood volume fraction, haemoglobin saturation, size of the scattering particles, density of the scattering particles and thickness. An additional variable parameter, a scale factor, was introduced to account for the adjustments of the normalization process where the measured reflectance spectra were normalized to a reflectance standard. This parameter is necessary because the spectra of the tissue and of reflectance standard were recorded from different probe-sample distances. There were therefore six parameters to be optimized.

Optimization was carried out using an adaptive approximate optimization method (Hidović and Rowe 2004), devised to overcome the long computation times involved in generating spectra using the Monte Carlo method. The speedup is obtained by finding a near-optimal solution using a fast but approximate model, in this instance Kubelka-Munk (Kubelka and Munk 1931), and then iteratively reducing the error between the approximate and the accurate model, i.e. Monte Carlo. The overall scheme is as follows:

1. Initially, set the error model to be zero.

2. Carry out optimization using Kubelka-Munk approximation (see below) and the error model.

3. Generate a spectrum from the parameters found in step 2 using Monte Carlo and probe geometry specific convolution. If the spectrum is deemed sufficiently close to the measured spectrum, terminate.

4. Run Monte Carlo on a number of parameter vectors close to the 'optimum' ones found in step 2.

5. Use these points to update the error model.

6. Go to step 2 .

This cycle is repeated until a sufficiently accurate spectrum is found, or until a maximum number of iterations is reached. At each cycle, the error model is corrected (step 5). This is done by sampling some points in the neighbourhood of the current optimum, and then making a linear approximation to the error. That is, we approximate the error between the Monte Carlo method and the current surrogate, by estimating its differential at the current optimum 

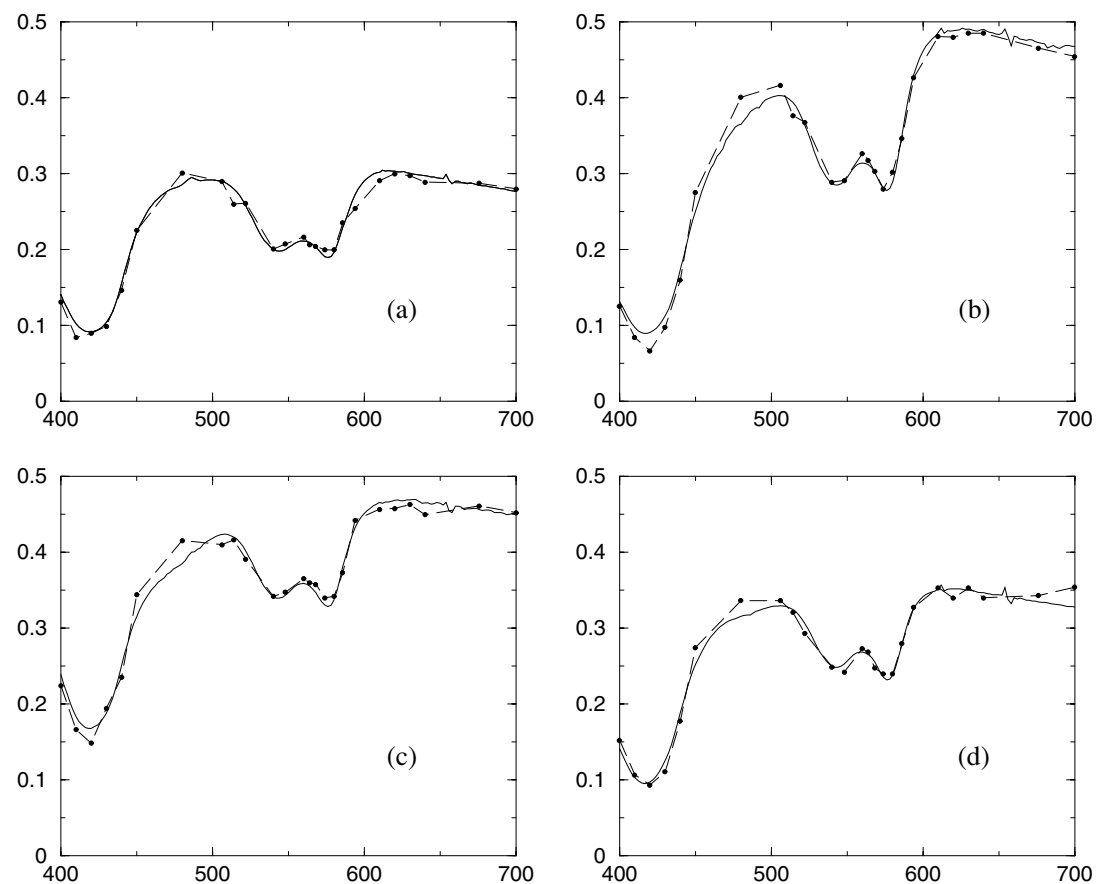

Figure 5. Typical results of the validation with the first set of data (Ge et al 1998). Simulated spectra (dash-dotted line) fit well the measured reflectance (solid line).

point. A detailed description of the method can be found in Hidović and Rowe (2004). The optimization to find the approximate solution in step 2 above uses an evolutionary strategy (Rowe and Hidović 2004).

\section{Results}

Figures 5 and 6 show typical results of the validation process. As can be seen, modelled and measured spectral reflectance curves agree well. This is particularly evident for the data collected by Ge et al (1998). In the validation against the second set of data (Sambongi et al 2002), small mismatches can be observed in the region between 400 and $450 \mathrm{~nm}$. Better fit in that region could have been obtained by taking into account inhomogeneous distribution of blood in the tissue (Amelink et al 2004, Verkruysee et al 1997, Finlay and Foster 2004). The slight missmatches between modelled and measured spectra could also be attributed to the nature of the optimization procedure described above. As the Monte Carlo method is computationally very expensive, the number of runs had to be limited rather than allowing the optimization to run its full course. For this reason the set of parameters found was not necessarily an optimal set, but a set deemed to be close enough, as measured by the distance function described by equation (6). Improved results can be produced by increasing the number of discrete wavelengths which represent a spectrum and by increasing the number of iterations, but at the cost of greatly increasing the computation time. The significant level of noise which can be observed in the measured spectra in the 400-450 nm region may have amplified the problem in that region. 

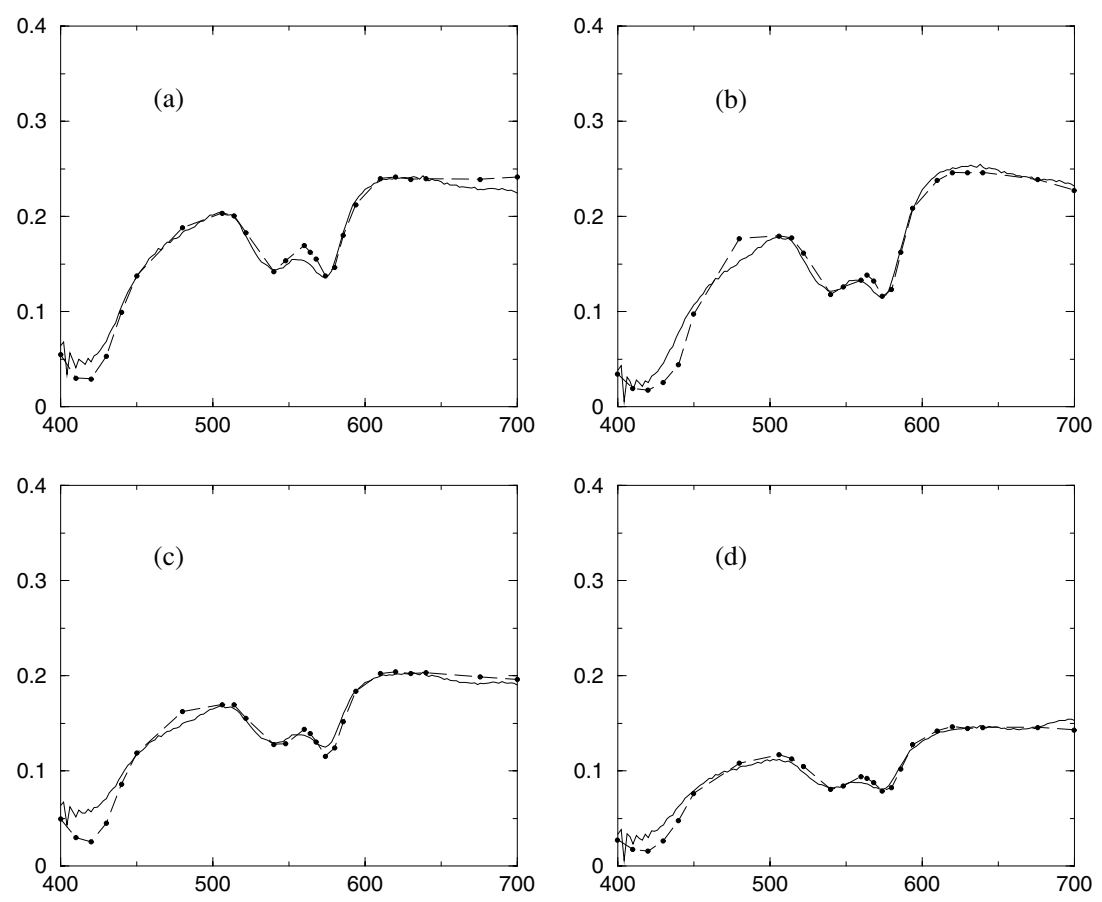

Figure 6. Typical results of the validation (dash-dotted line) with the second set of data (Sambongi et al 2002) (solid line).

Table 2. Mean and standard deviation of mucosal parameter values corresponding to the matched model curves. The values are in the histologicaly plausible ranges.

\begin{tabular}{llllrr}
\hline & $\begin{array}{l}\text { Volume fraction } \\
\text { of blood }\end{array}$ & $\begin{array}{l}\text { Haemoglobin } \\
\text { saturation }\end{array}$ & $\begin{array}{l}\text { Mean size of } \\
\text { scattering particles }\end{array}$ & $\begin{array}{l}\text { Mean volume fraction } \\
\text { of scattering particles }\end{array}$ & $\begin{array}{r}\text { Thickness } \\
\text { Mean }\end{array}$ \\
Standard deviation & $2.50 \%$ & $70.3 \%$ & $0.3365 \mu \mathrm{m}$ & $16.80 \%$ & $446 \mu \mathrm{m}$ \\
\hline
\end{tabular}

A quantitative error analysis was performed on the set of 54 spectra of the normal colon tissue (Ge et al 1998) referred to in section 5. The mean error ( \pm STD) of the difference between the curves, calculated using equation (6), was $0.0104( \pm 0.0037)$. The extracted parameter values are shown in table 2 .

The comparison of the reflectance curves obtained using different probe geometries has shown that different measurement methods produce reflectance spectra of different magnitudes and shapes. This can be observed in figures 5 and 6. The data provided by Ge et al (1998) was acquired with a probe placed in contact with the tissue. As a result, only a small fraction of photons in the red region were collected, as they travel larger paths inside the tissue, and hence re-emerge at the tissue surface at large source to collection distances. The noncontact probe, on the other hand, generally collects photons from a slightly larger area on the tissue surface than that of the probe itself. This results in an increased number of red photons that get collected compared to photons collected by a contact probe of the same diameter. 


\section{Discussion and future work}

This paper has described the structure and the parametrization of an optical model of the colon. Verification has demonstrated that the model is able to generate spectra which approximate well the spectra measured in real colon tissue. However, the model would benefit from a number of improvements, the most important of which are to implement more realistic models of scattering in the tissue, and to refine the absorption coefficient for blood to take into account its inhomogeneous distribution and the effects of the vessel size. Discussion in section 7.1 addresses these issues.

As we have seen, the validation process was carried out only on the normal spectra, as the model developed so far represents only the normal colon tissue. Clearly, to be of diagnostic use, the model needs to account for pathological changes. We have carried out some preliminary work which indicates that by modifying the parameters of the current model, so that they reflect histopathological changes associated with colon cancer, we can obtain spectra resembling measured spectral reflectance of adenocarcinomas. This is discussed in section 7.2.

The most important aspect of our future work is the diagnostic interpretation of the measured data. Quantitative parameters obtained from the optimization method used for the model validation (section 5) could potentially be used to characterize the mucosal tissue. However, this method is time consuming and applicable only to single-point full spectral data. In section 7.3 we outline the proposal for deriving parameters from multi-spectral images comprising a small number of spectral bands. In the proposed approach, image data would be acquired using a modified endoscope, interpretation would be much more rapid, and parameter values would be presented in the form of images showing locations and spatial patterns of any abnormal blood and collagen features. This method of interpretation has already been successfully implemented for the skin (Claridge et al 2003), and as a result, a system able to assist clinicians in diagnosis of melanoma was developed (Moncrieff et al 2002).

It is important to stress that the interpretation of the spectra based on the physics of light interaction with tissue differs significantly from traditional approaches to spectral analysis in which statistical or pattern analysis techniques are applied to the spectra, without considering the process of their formation. Such analysis, with the aim of distinguishing the neoplastic and non-neoplastic tissues, was carried out by a number of authors, for example Mourant et al (1996), Ge et al (1998), Sambongi et al (2002). In contrast to the above technique, a physics-based approach relies on a fundamental understanding of the formation process of spectra, which enables it to provide a histological interpretation of spectral features, rather than just statistically analysing them.

\subsection{Possible ways of improving the model}

The scattering properties of the colon layers were modelled by taking into account the physical properties of collagen, and assuming the homogeneity of each layer. A possible shortcoming of this simplification is that the parameters returned from fitting to the model may not correctly reflect histopathological changes. The model could clearly be refined by considering each of the scattering components as a separate entity, each with its representative range of parameter values. This is a worthwhile objective because there is growing evidence that the sizes and densities of scattering particles, including subcellular organelles and chromatin-rich cells, affect the spectral and angular distribution of polarized light and produce diagnostically important differences in the remitted spectra (Backman et al 2000, Gurjar et al 2001, Fang et al 2003). 
Further improvement in modelling of scattering may result from considering the nonspherical nature of the scattering particles. However, recent work by Chen et al (2004) suggests that the modelling of non-spherical particles as spheres can yield acceptable scattering coefficient. That paper introduces the concept of the equisphere, which is a sphere that gives rise to the maximum phase shift of the non-spherical particle of interest. It is shown then that the scattering cross-section of non-spherical particles has the same periodic characteristics as the cross-section of their spherical counterparts, with some differences in the amplitude. These differences can be compensated for when multiplying the scattering cross-section by the density of the scattering particles in order to get the scattering coefficient. Our model varies the scattering cross-section and the particle density separately to arrive at the optimal scattering coefficient for a given spectrum. Thus, taken individually, each of the variables may show a small error; however, on the strength of the work by Chen et al (2004), their product is likely to be correct. Whilst this approach is deemed to be satisfactory for our current model, we intend to work towards a more detailed model of scatter for non-spherical particles such as collagen, and subcellular organelles.

Another potential improvement concerns the modelling of the absorption coefficient. The expression for blood absorption (3) used in this work is based on the measurement of haemoglobins in a homogeneous solution. In most real tissues blood is confined to blood vessels which occupy only a small portion of the tissue volume. Recent experimental work by Van Veen et al (2002), Amelink et al (2004) and Finlay and Foster (2004) demonstrates that such an inhomogeneous distribution of blood pigments may significantly affect the blood absorption coefficient. Van Veen et al (2002) and Amelink et al (2004) suggest a correction factor which is approximately inversely proportional to the product of $\mu_{a}$ of whole blood and the size of the vessel diameter. The effect of the correction is primarily to flatten the spectral absorption curve in the Soret band, with only small effect on the wavelengths above $450 \mathrm{~nm}$. The differences between the measured spectra and our model-derived reflectance values in $400-450 \mathrm{~nm}$ band in figures 5 and 6 can be attributed to the lack of the correction factor in the current version of our model. In the future imaging work, discussed in section 7.3, we are unlikely to acquire images of the colon in the range below $450 \mathrm{~nm}$. In this part of the spectrum standard CCD camera chips have relatively low quantum efficiency, which gives rise to an unfavourable signal to noise ratio. However, in general, the reflectance in the Soret band is important in tissue studies and the incorporation of the correction factor is recommended.

\subsection{Reflectance of cancerous tissue: preliminary findings}

For the method to be applicable to a diagnostic investigation of the colon, histological parameters which affect the spectral reflectance should differ between normal and abnormal tissue. Our preliminary investigation indicates that this indeed may be the case. It is well known that tumour cells grow and divide very quickly. Growth of the tumour depends on the growth and density of the supporting blood vessels. Skinner et al (1995) studied microvascular structure of tumours in human colon. They found that the blood vessels within carcinomas were characterized by disorganization, increased size and density compared to normal colon. Increased vasculature implies increased blood flow. This finding was supported by Zografos et al (1990), who measured blood flow by the laser Doppler flowmetry and found that it increased in human rectal carcinomas when compared to normal colonic mucosa. Haemoglobin saturation is also known to vary between normal and abnormal tissue. In particular, Ge et al (1998) reported a decrease in oxygen saturation in adenocarcinoma of colon. Hilska et al (1998) have studied the distribution of collagen in normal and malignant colorectal mucosal tissue and observed that both the epithelium basement membrane (composed mainly 

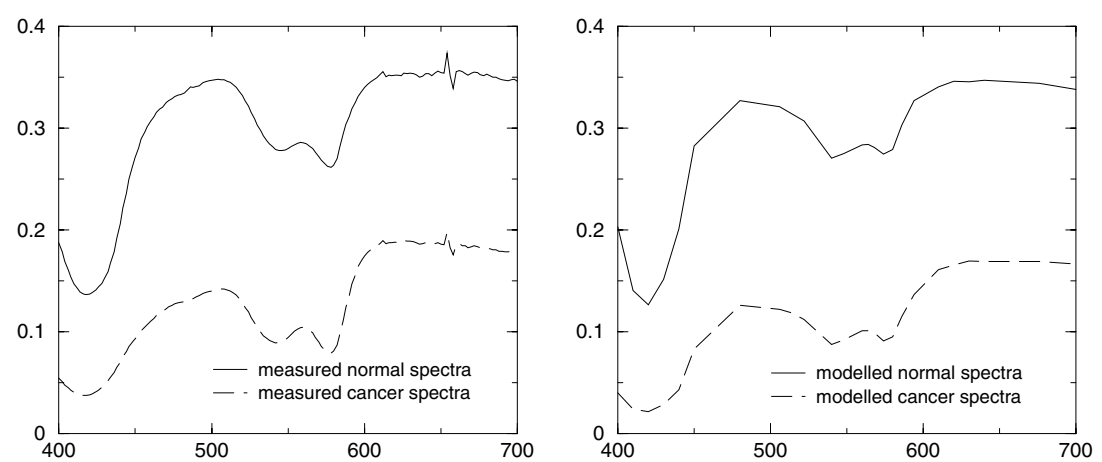

Figure 7. Left: the measured spectra of normal (solid line) and cancerous (dashed line) tissue. Right: the relative modelled spectra (see the text for the explanation).

of collagen IV) and the collagenous matrix beneath it (composed of collagen type I and III) were disturbed in the malignant tissue. Even if unable to identify the stage or grade of tumour, their studies showed that loss of the basement membrane in colorectal carcinoma increases the probability of distant metastases. Furuya and Ogata (1993) studied the collagen network of the colon by means of electron microscopy. Their main findings suggested an increase in the density of collagen in the central region of the cancer whereas looser networks of thinner collagen fibrils were observed towards the margin of the tumour. Furthermore, Turnay et al (1989) studied collagen metabolism in human colon adenocarcinoma. Their main findings suggest an increase in the collagen content in the first stages of the neoplasia, followed by a decrease during the development of the tumour, with the overall collagen content being lower in the tumour.

With the aim of demonstrating the ability of the model to generate abnormal spectra we have conducted a computational experiment in which the ranges of parameter values in the model were changed to correspond to the changes in the colon tissue discussed above. Figure 7 shows graphs of measured and modelled spectra of normal and cancerous tissue. The graph on the left shows the spectrum measured in vivo in a cancerous and an adjacent normal tissue of a patient Ge et al (1998). The graph on the right shows the modelled spectra. The top graph (solid line) is a spectrum of a normal colon obtained during the validation process described in section 4. Parameter values extracted were in the histologically plausible ranges (table 3). The bottom graph (dashed line) shows a spectrum derived from the normal one above. It was generated by altering the parameters for the blood and collagen content in the mucosa in line with the changes in the tissue structure which occur in cancer, as discussed above. In particular, the blood volume fraction and the thickness of the mucosa were increased, while collagen content was decreased (table 3). As can be seen, this spectrum resembles the measured spectrum of abnormal tissue, adenocarcinoma, shown on the left.

It should be stressed that the above experiment does not represent the verification of the model for abnormal spectra, but just a demonstration that the model is able to generate abnormal spectra when the values of histological parameters are changed to reflect the histological changes associated with the abnormal tissue, as discussed above. More detailed study is currently being carried out in order to understand how the tissue abnormalities influence the final remittance. However, on the strength of the arguments that, first, a developing cancer changes the characteristics of blood and collagen in the mucosa, and second, that changes in both these factors have significant effect on the remitted light in normal tissue, we expect that the shapes of the spectra for abnormal tissue will be affected in a characteristic way. If normal 
Table 3. Parameter values used to generate spectra in figure 7.

\begin{tabular}{llllll}
\hline $\begin{array}{l}\text { Tissue } \\
\text { type }\end{array}$ & $\begin{array}{l}\text { Volume fraction } \\
\text { of blood }\end{array}$ & $\begin{array}{l}\text { Haemoglobin } \\
\text { saturation }\end{array}$ & $\begin{array}{l}\text { Mean size of } \\
\text { scattering particles }\end{array}$ & $\begin{array}{l}\text { Volume fraction of } \\
\text { scattering particles }\end{array}$ & Thickness \\
\hline Normal & $1.985 \%$ & $70 \%$ & $0.2665 \mu \mathrm{m}$ & $9.9 \%$ & $532 \mu \mathrm{m}$ \\
Cancer & $6.150 \%$ & $70 \%$ & $0.2665 \mu \mathrm{m}$ & $5.0 \%$ & $632 \mu \mathrm{m}$ \\
\hline
\end{tabular}

and abnormal spectra are shown to exhibit characteristic patterns and behaviours, they can be used to characterize colon pathologies, including colon cancer.

\subsection{Multi-spectral imaging and interpretation to aid diagnosis}

One of the important challenges in the fight against colon cancer is the detection of early changes (Gurjar et al 2001, Wax et al 2002) which may not be apparent in endoscopic images. A disadvantage of single-point spectroscopy is, in this respect, that a probe operator may miss a potentially suspicious site. An ideal solution would be a hyperspectral image which registers a whole spectrum at each image point. Although such a technology exists, it normally requires long acquisition times which are not feasible for in vivo imaging of the colon.

Our earlier work has shown that under certain conditions it is possible to recover parameter values relatively accurately from a small number of optimally selected spectral bands (Claridge and Preece 2003). Four parameters characterizing the mucosa (thickness, blood volume fraction, size and density of collagen fibrils-see section 4) require four bandpass filters, making the image acquisition with the existing endoscope technology feasible.

The relationship between the multi-spectral image vectors and the parameters can be derived from the spectral reflectance model described in this paper as follows. By varying the model parameters across the whole range of their plausible values (section 3.2), reflectance spectra corresponding to all normal colon tissues can be generated. Next, by convolving the spectra with the four bandpass filters selected through optimization, all plausible fourdimensional image vectors can be computed. In this way the cross-reference between the tissue parameters and the image vectors is established. Provided that the mapping between the parameters and image vectors is unique (Claridge and Preece 2003), parameter values can be derived from the measured four-band image data. Information about the histological parameters characterizing the colon can then be represented in a form of grey-scale images with each pixel showing the magnitude of the respective parameter. This should permit the reconstruction of the structure of colon tissue and its further analysis.

\section{Conclusions}

An optical model of the colon has been developed which, using Monte Carlo simulations, permits the calculation of the spectral reflectance of colon tissue for ranges of histological parameters. The validation process has shown that the model is able to generate spectra which correspond to measured spectral reflectance of colon tissue. Moreover, the ranges of parameters used in the model are histologically plausible, which lends weight to our assertion that the model is correct. As part of the model development, the parameters characterizing the optical and physical properties of the colon have been established and brought together for the first time. The effect of the individual parameters on the colon spectra was analysed, enabling fresh understanding of the effect of the colon structure on the remitted spectrum. The analysis resulted in two particularly useful conclusions. First, the modelling experiments 
have shown that the remitted light is affected mainly by the structure and contents of mucosa, and that the tissues lying further into the light path have a small, but constant effect. This has allowed us to reduce the number of variable parameters from nine to four and thus to considerably simplify the model. Second, we have observed that the variations in each of the four parameters affect the shapes of the spectra in different combinations of spectral regions. This behaviour suggests that it may be possible to carry out the model 'inversion' and to recover parameters characterizing the colon from images acquired through optimally selected filters. It is hoped that in the long term the results of this research will be used to help with the diagnosis of colon disorders, and in particular colon cancer.

\section{Acknowledgments}

Kevin Schomacker (MediSpectra, Inc) and Kazuhiro Gono (Olympus) are gratefully acknowledged for providing us with in vivo spectral reflectances of colon tissue, and Jon Rowe (University of Birmingham) for his help and guidance, especially with optimization algorithms. We would also like to thank Mr Tariq Ismail (University Hospital Birmingham) and Dr Nigel Suggett (University of Birmingham) for their help with endoscopic images of colon, and Dr Scott Sanders (Warwick Hospital) for validating the histological plausibility of the model and its parameters. We also wish to thank the anonymous referees for their valuable comments and suggestions.

\section{References}

Amelink A, Sterenborg H, Bard M and Burgers S 2004 In vivo measurement of the local optical properties of tissue by use of differential path-length spectroscopy Opt. Lett. 29 1087-9

Backman V et al 2000 Detection of preinvasive cancer cells in situ Nature 406 35-6

Badizadegan K et al 2004 Spectroscopic diagnosis and imaging of invisible pre-cancer Faraday Discuss. 126 265-79

Beek J F, Blokland P, Posthumus P, Aalders M, Pickering J W, Sterenborg H J C and Van Gemert M J C 1997 In vitro double-integrating-sphere optical properties of tissues between 630 and $1064 \mathrm{~nm}$ Phys. Med. Biol. 42 2255-61

Bohren C and Huffman D R 1983 Absorption and Scattering of Light by Small Particles (New York: Wiley)

Chen Z, Taflove A and Backman V 2004 Concept of the equiphase sphere for light scattering by nonspherical dielectric particles J. Opt. Soc. Am. A 21 88-97

Claridge E, Cotton S, Hall P and Moncrieff M 2003 From colour to tissue histology: Physics based interpretation of images of pigmented skin lesions Med. Image Anal. 7 489-502

Claridge E and Preece S J 2003 An inverse method for the recovery of tissue parameters from colour images Information Processing in Medical Imaging vol LNCS 2732 ed C Taylor and J A Noble (Berlin: Springer) 306-17

Cotton S D and Claridge E 1996 Developing a predictive model of human skin colouring ed R Vanmetter and J Beutel Proc. SPIE Med. Imaging 2708 814-25

Dacosta R S, Wilson B C and Marcon N E 2002 New optical technologies for earlier endoscopic diagnosis of premalignant gastrointestinal lesions J. Gastroen. Hepatol. 17 85-104

Delori F C and Pflibsen K P 1989 Spectral reflectance of the ocular fundus Appl. Opt. 28 1061-77

Fang H et al 2003 Noninvasive sizing of subcellular organelles with light scattering spectroscopy IEEE J. Sel. Top. Quant. 9 267-76

Farrell T J, Patterson M S and Wilson B C 1992 A diffusion theory model of spatially resolved, steady-state diffuse reflectance for the non-invasive determination of tissue optical properties Med. Phys. 19 879-88

Finlay J C and Foster T H 2004 Effect of pigment packaging on diffuse reflectance spectroscopy of samples containing red blood cells Opt. Lett. 29 965-7

Friedland S, Benaron D, Parachikov I and Sotikno R 2003 Measurement of mucosal capillary hemoglobin oxygen saturation in the colon by reflectance spectrophotometry Gastrointest. Endosc. 57 492-7

Furuya Y and Ogata T 1993 Scanning electron microscopic study of the collagen networks of the normal mucosa, hyperplastic polyp, tubular adenoma and adenocarcinoma of the human large intestine Tohoku J. Exp. Med. 169 $1-19$ 
Ge Z, Schomacker K T and Nishioka N S 1998 Identification of colonic dysplasia and neoplasia by diffuse reflectance spectroscopy and pattern recognition techniques Appl. Spectrosc. 52 833-9

Georgakoudi I, Motz J T, Backman V, Anghelou G, Haka A S, Muller M, Dasari R and Feld M S 2003 Quantitative characterization of biological tissue using optical spectroscopy Biomedical Photonics Handbook ed T Vo-Dinh (Boca Ratan, FL: CRC Press) chapter 31

Gurjar R S, Backman V, Perelman L T, Georgakoudi I, Badizadegan K, Itzkan I, Dasari R R and Feld M S 2001 Imaging human epithelial properties with polarised light-scattering spectroscopy Nat. Med. 7 1245-8

Hajivassiliou C A, Greer K, Fisher A and Finlay I G 1998 Non-invasive measurement of colonic blood flow distribution using laser Doppler imaging Brit. J. Surg. 85 52-5

Hammer M and Schweitzer D 2002 Quantitative reflection spectroscopy at the human ocular fundus Phys. Med. Biol. 47 179-91

Hidović D and Rowe J E 2004 Validating a physics-based model of colon colouration using an adaptive approximate optimisation method Proc. GECCO 2004 (Lecture Notes in Computer Science vol 3103) ed K Deb et al (Berlin: Springer) 1005-16

Hilska M, Collan Y, Peltonen J, Gullichsen R, Paajanen H and Laato M 1998 The distribution of collagen types I, III and IV in normal and malignant colorectal mucosa Eur. J. Surg. 164 457-64

Huang Z, Zheng W, Xie S, Chen R, Zeng H, McLean D I and Lui H 2004 Laser-induced autoflorescence microscopy of normal and tumor human colonic tissue Int. J. Oncol. 24 59-63

Jacques S L 1996 Origins of tissue optical properties in the UVA, visible and NIR regions OSA Trends opt.photon Adv. Opt. Imaging Photon Migration 2 364-71

Jacques S L 1998 Skin Optics (Portland, OR: Oregon Medical Laser Centre) http://omlc.ogi.edu/news/jan98/ skinoptics.html

Jacques S L, Bargo P and Engelking K 2003 Optical fiber reflectance spectroscopy Saratov Fall Meeting http://optics.sgu.ru/SFM/2003/internet/sjacques/index.html

Kubelka P and Munk F 1931 Ein Beitrag zur Optik der Farbanstriche Z. Tech. Physik 12 593-601

Marchesini R, Bertoni A, Andreola S, Melloni E and Schirollo A E 1989 Extinction and absorption coefficients and scattering functions in human tissues in vitro Appl. Opt. 28 2318-24

Marchesini R, Pignoli E, Tomatis S, Fumagali S, Sichrollo A E, Di Palma S, Dal Fante M, Spinelli P, Croce A C and Battioli G 1994 Ex vivo optical properties of human colon tissue Lasers Surg. Med. 351-7

Meglinski I V and Matcher S J 2002 Quantitative assessment of skin layers absorption and skin reflectance spectra simulation in the visible and near-infrared spectral regions Physiol. Meas. 23 741-53

Moncrieff M, Cotton S, Claridge E and Hall P 2002 Spectrophotometric intracutaneous analysis: a new technique for imaging pigmented skin lesions Brit. J. Dermatol. 146 1-11

Mourant J R and Bigio I J 2003 Elastic-scattering spectroscopy and diffuse reflectance Biomedical Photonics Handbook ed T Vo-Dinh (Boca Ratan, FL: CRC Press) chapter 29

Mourant J R, Bigio I J, Boyer J, Johnson T M, Lacey J, Bohorfoush A G and Mellow M 1996 Elastic scattering specroscopy as a diagnostic tool for differentiating pathologies in gastrointestinal tract: preliminary testing J. Biomed. Opt. 1 192-9

Mourant J R, Fuselier T, Boyer J, Johnson T M and Bigio I J 1997 Predictions and measurement of scattering and absorption over broad wavelength ranges in tissue phantoms Appl. Opt. 36 949-57

Perelman L T et al 1998 Observation of periodic fine structure in reflectance from biological tissue: a new technique for measuring nuclear size distribution Phys. Rev. Lett. 80 627-30

Prahl S A 1999 Optical Absorption of Hemoglobin (Portland, OR: Oregon Medical Laser Centre) http:// omlc.ogi.edu/spectra/hemoglobin/index.html

Prahl S A 2000 Mie Theory (Portland, OR: Oregon Medical Laser Centre) http://omlc.ogi.edu/software/mie/

Preece S J and Claridge E 2002 Monte Carlo modelling of the spectral reflectance of the human eye Phys. Med. Biol. 47 2863-77

Rowe J E and Hidović D 2004 An evolution strategy using a continuous version of the Gray-code neighbourhood distribution Proc. GECCO 2004 (Lecture Notes in Computer Science vol 3102) ed K Deb (Berlin: Springer) 725-36

Sambongi M, Igarashi M, Obi T, Yamaguchi M, Ohyama N, Kobayashi M, Sano Y, Yoshida S and Gono K 2002 Analysis of spectral reflectance using normalisation method from endoscopic spectroscopy system Opt. Rev. 9 238-43

Skinner S A, Frydman G M and O'Brien P E 1995 Microvascular structure of benign and malignant tumors of the colon in humans Dig. Dis. Sci. 40 373-84

Skinner S A and O'Brien P E 1996 The microvascular structure of normal colon in rats and humans J. Surg. Res. 61 482-90 
Thomson H J, Busuttil A, Eastwood M A, Smith A N and Elton R A 1986 The submucosa of the human colon $J$. Ultrastruct. Mol. Struct. Res. 96 22-30

Tuchin V V 2000 Tissue Optics: Light Scattering Methods and Instruments for Medical Diagnosis (Tutorial Texts in Optical Engineering Series vol TT38) 1st edn (Bellingham,WA: SPIE-International Society for Optical Engineering)

Turnay J, Olmo N, Gavailanes J G and Lizarbe M A 1989 Collagen metabolism in human colon adenocarcinoma Conn. Tiss. Res. 23 251-60

Van Veen R, Werkruysse W and Sterenborg H 2002 Diffuse-reflectance spectroscopy from 500 to $1060 \mathrm{~nm}$ by correction for inhomogeneously distributed absorbers Opt. Lett. 27 246-8

Verkruysee W, Lucassen G W, de Boer J F, Smithies D J, Nelson J S and van Gemert M J C 1997 Modelling light distributions of homogeneous versus discrete absorbers in light irradiated turbid media Phys. Med. Biol. 42 $51-65$

Wang L H, Jacques S L and Zheng L Q 1995 MCML-Monte Carlo modeling of photon transport in multi-layered tissues Comput. Meth. Progr. Biomed. 47 131-46

Wang L H, Jacques S L and Zheng L Q 1997 CONV_Convolution for responses to a finite diameter photon beam incident on multilayered tissues Comput. Meth. Progr. Biomed. 54 141-50

Wax A, Yang C, Backman V, Badizadegan K, Boone C W, Dasari R R and Feld M S 2002 Cellular organization and substructure measured using angle-resolved low-coherence interferometry Biophys. J. 82 2256-64

Zangaro R A, Silveira L, Manoharan R, Zonios G, Itzkan I, Dasari R R, Van Dam J and Field M S 1996 Rapid multiexcitation fluorescence spectroscopy system for in vivo tissue diagnosis Appl. Opt. 35 5211-9

Zografos G C, Iftikhar S Y, Harrison J and Morris D L 1990 Evaluation of blood flow in human rectal tumors using a laser Doppler flowmeter Eur. J. Surg. Oncol. 16 497-9

Zonios G, Cothren R M, Arendt J T, VanDam J, Crawford J M and Manoharan R 1996 Morphological model of human colon tissue fluorescence IEEE Tron. Biomed. Eng. 43 113-22

Zonios G, Perelman L T, Backman V, Manoharan R, Fitzmaurice M, Dam J V and Feld M S 1999 Diffuse reflectance spectroscopy of human adenomatous colon polyps in vivo Appl. Opt. 38 6628-37 